\title{
Capacity Scaling Laws for Underwater Networks
}

\author{
Daniel E. Lucani, Muriel Médard, and Milica Stojanovic
}

Abstract. The underwater acoustic channel is characterized by a path loss that depends not only on the transmission distance, but also on signal frequency. Signals transmitted from one user to another over a distance $l$ are subject to a power loss of $l^{-\alpha} a(f)^{-l}$. Although a terrestrial radio channel can be modeled similarly, the underwater acoustic channel has different characteristics. The spreading factor $\alpha$, related to the geometry of propagation, has values in the range $1 \leq \alpha \leq 2$. The absorption coefficient $a(f)$ is a rapidly increasing function of frequency: it is three orders of magnitude greater at $100 \mathrm{kHz}$ than at a few hertz. Existing results for capacity of radio wireless networks correspond to scenarios for which $a(f)=1$, or a constant greater than one, and $\alpha \geq 2$. These results cannot be applied to underwater acoustic networks in which the attenuation varies over the system bandwidth. We use a water-filling argument to assess the minimal transmission power and optimal transmission band as functions of the link distance and desired data rate, and study the capacity scaling laws under this model. We show that the transport capacity increases at most at a rate $n^{1-1 / \alpha} e^{-W_{0}\left(O\left(n^{-1 / \alpha}\right)\right)}$, where $W_{0}$ represents the branch zero of the Lambert $\mathrm{W}$ function, for the cases in which the transmission band is either fixed a priori or assigned in relation to the transmission distance. This means that the transport capacity increases much more slowly than that of multihop routing in wireless scenarios, which is bounded by $O\left(n^{1 / 2}\right)$. Finally, we show that scaling the frequency of the transmission band with the number of nodes provides a means to exploit characteristics of the acoustic channel in order to overcome the previous pessimistic results.

(C) Taylor \& Francis Group, LLC

ISSN: I542-795I print 


\section{Introduction}

The seminal work [Gupta and Kumar 00] studied wireless networks, modeled as a set of $n$ nodes that exchange information, with the aim of determining what amount of information the source nodes can send to the destination as the number $n$ grows. The original results obtained for nodes deployed in a disk of unit area motivated the study of capacity-scaling laws in different scenarios, ranging from achievability results in random deployments using percolation theory [Franceschetti et al. 07] and cooperation between nodes [Ozgur et al. 07] to the impact of node mobility on the capacity of the network, e.g., [Grossglauser and Tse 02]. A good overview of the different assumptions and scaling laws for radio wireless networks is provided by [ $\mathrm{Vu}$ et al. 08].

The underwater acoustic channel is characterized by a path loss that depends not only on the transmission distance, but also on the signal frequency [Stojanovic 07]. Signals transmitted over a distance $l$ are subject to a power loss of $l^{-\alpha} a(f)^{-l}$. Although a terrestrial radio channel can be modeled similarly, the underwater acoustic channel has important differences. The spreading factor $\alpha$, related to the geometry of propagation, has values in the range $1 \leq \alpha \leq 2$, where $\alpha=1$ corresponds to cylindrical spreading and $\alpha=2$ to spherical spreading. Also, the absorption coefficient $a(f)$ is a rapidly increasing function of frequency, e.g., it is three orders of magnitude greater at $100 \mathrm{kHz}$ than at a few hertz [Stojanovic 07]. Finally, the background noise is not white, but has a power spectral density that is highly dependent on frequency.

Existing capacity-scaling laws for wireless radio networks correspond to scenarios for which $a(f)=1$, or a constant greater than one, and $\alpha \geq 2$, e.g., [Gupta and Kumar 00, Franceschetti et al. 07]. These results cannot be directly applied to underwater acoustic networks in which the attenuation varies over the system bandwidth and $\alpha \leq 2$.

We study the scaling laws under a model that considers a water-filling argument to assess the minimal transmission power and optimal transmission band as functions of the link distance and desired data rate [Lucani et al. 08b]. In particular, we study the case of arbitrarily deployed networks in a disk of unit area, and follow a similar procedure as in [Gupta and Kumar 00] to derive an upper bound on the capacity. In this sense, we provide an extension of the work in [Gupta and Kumar 00] under a more complicated power-loss model.

Seeking to characterize the fundamental capacity scaling of underwater acoustic networks in a dense network scenario, we show that the amount of information that can be exchanged by each source-destination pair in an underwater acoustic network goes to zero as the number of nodes $n$ goes to infinity. This occurs at a 
rate at least

$$
n^{-1 / \alpha} e^{-W_{0}\left(O\left(n^{-1 / \alpha}\right)\right)}
$$

where $W_{0}$ represents the branch zero of the Lambert $\mathrm{W}$ function [ChapeauBlondeau and Monir 02]. ${ }^{1}$ We illustrate that this throughput has two different regions per source-destination pair. For small $n$, the throughput decreases very slowly as $n$ increases. For large $n$, it decreases almost as $n^{-1 / \alpha}$. Thus, for large enough $n$, the throughput decreases more rapidly in underwater networks than in typical radio networks, because of the difference in the path-loss exponent $\alpha$. Finally, we show that scaling the frequency of the transmission band with the number of nodes provides a means to exploit characteristics of the acoustic channel in order to overcome the previous pessimistic results. In particular, we study the effect of scaling the transmission frequency with $n$ on our upper bound and provide a constructive lower bound that overcomes the limitations observed for a fixed transmission band. Note that for a fixed transmission band, the transport capacity of the network increases much more slowly than that of multihop routing in wireless scenarios, i.e., much more slowly than $O\left(n^{1 / 2}\right)$.

The paper is organized as follows. In Section 2, we present the underwater channel model. In Section 3, we analyze the scaling laws for the case of a network transmitting in an arbitrarily chosen narrow band and propose constructive schemes to support our findings. In Section 4, we study scaling laws for the lowpower/narrow-band case, with optimal bandwidth allocation using a water-filling argument and also consider the case in which the nodes can transmit at high power over a wide transmission band. Section 5 studies the effect of scaling the transmission frequency with the number of nodes. Conclusions are summarized in Section 6.

\section{Underwater Channel Model}

An underwater acoustic channel is characterized by an attenuation that depends on the distance $l$ and the signal frequency $f$ as

$$
A(l, f)=\left(\frac{l}{l_{\mathrm{ref}}}\right)^{\alpha} a(f)^{l-l_{\mathrm{ref}}},
$$

\footnotetext{
${ }^{1}$ We use the following notation: (i) $f(x)=O(g(x))$ means that there exists a constant $C$ such that $x \rightarrow \infty \lim f(x) / g(x)=C$, (ii) $f(x)=o(g(x))$ means that $x \rightarrow \infty \lim f(x) g(x)=0$, and (iii) $f(x)=\Omega(g(x))$ if $g(x)=o(f(x))$.
} 


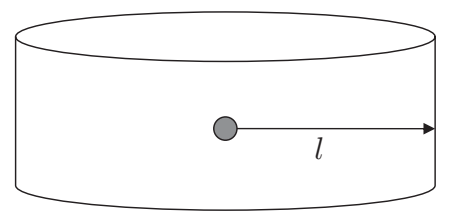

(a)

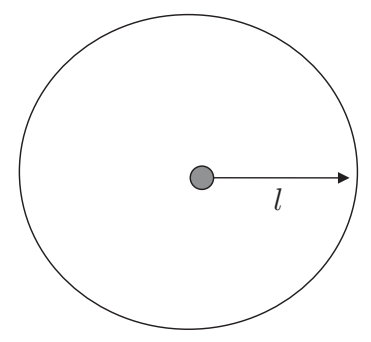

(b)

Figure I. Spreading geometries in underwater communications: (a) cylindrical, $\alpha=1$; (b) spherical $\alpha=2$.

where $l_{\text {ref }}$ is a reference distance (typically $1 \mathrm{~m}$ ). Note that this model generalizes the free-space model.

A common empirical model used for the absorption $a(f)$ is Thorp's formula, which for $f$ in kilohertz is given by [Stojanovic 07]

$$
10 \log a(f)=0.11 \frac{f^{2}}{1+f^{2}}+44 \frac{f^{2}}{4100+f^{2}}+2.75 \cdot 10^{-4} f^{2}+0.003
$$

This absorption $a(f)$ is a strictly increasing function of $f$. Note that $a(f)$ in underwater channels is typically defined with respect to a specific reference distance $l_{a}$ (usually $1 \mathrm{~km}$ ). Thus, our $a(f)$ can be thought of as $a(f)=a_{n}(f)^{1 / l_{a}}$, where $a_{n}(f)$ is a normalized absorption parameter. We emphasize that $a(f)^{l}=$ $a_{n}(f)^{l / l_{a}}$, where $l / l_{a}$ is clearly unit-free.

The spreading factor describes the geometry of propagation and is typically $1 \leq \alpha \leq 2$, e.g., $\alpha=1$ and $\alpha=2$ correspond to cylindrical and spherical spreading, respectively. A cylindrical spreading, Figure 1(a), corresponds to cases in which the transmission distance $l$ is much larger than the depth of the ocean. In this case, the ocean bottom and the interface between the ocean and the air act as boundaries for the spreading of acoustic waves. This problem can be modeled as a cylindrical wave guide. On the other hand, spherical spreading, Figure 1(b), is considered when the transmission distance is smaller than the depth of the ocean. This type of spreading provides a similar $\alpha$ to that of the free-space approximation for radio wireless communications. For the case of nearfield transmissions, the value of $\alpha$ depends on the type of sound source. Two main types have been identified, namely the monopole and dipole with associated $\alpha$ of 2 and 4, respectively [Bass et al. 03].

The noise in an acoustic channel can be modeled through four basic sources: turbulence, thermal noise, shipping, and waves. It has a power spectral density 
(psd) that depends on the frequency, the shipping activity $s$, and the wind speed $w$ in meters per second [Stojanovic 07].

The complete model for a colored Gaussian underwater link was presented in [Lucani et al. 08b], where power was allocated through water-filling. In the absence of multipath and channel fading, the relationship among capacity, transmission power, and optimal transmission band of a point-to-point link is given by [Lucani et al. 08b]

$$
C=\int_{B(l, C)} \log _{2}\left(\frac{K(l, C)}{A(l, f) N(f)}\right) d f,
$$

where $N(f)$ is the psd of the noise, $B(l, C)$ is the optimum band of operation, and $K(l, C)$ is a constant whose value is determined by the link distance $l$ and the desired capacity $C$. The transmission power associated with a particular choice of $(l, C)$ is given by

$$
P(l, C)=\int_{B(l, C)} S(l, C, f) d f
$$

where the psd of the signal is $S(l, C, f)=K(l, C)-A(l, f) N(f), f \in B(l, C)$.

A distinguishing feature of the underwater acoustic channel is the dependence of the optimal transmission band on the link distance [Lucani et al. 08b]. Figure 1 illustrates the optimal center frequency $f_{c}(l)$ as a function of distance. The optimal center frequency is defined as the frequency at which $A(l, f) N(f)$ is minimal. This implies that if the transmission power for a link is low, the transmission bandwidth will be low and around the optimal frequency. Thus, the optimal transmission band in the spectrum changes dramatically with the link distance. Figure 2 also illustrates that a node transmitting over a short range will optimally be assigned a transmission band at high center frequency, as in case (a), while a node transmitting over a longer distance will be assigned a different transmission band at lower center frequency, as in case (b).

For the case in which the power available for transmission is low, the bandwidth of the transmission band will also be small. When the bandwidth is low enough, $|B(l, C)|=\Delta f$, such that the product $A(l, f) N(f)$ does not change much over that band, one can take a Taylor series approximation around the center frequency $f_{c}(l)$. This allows us to determine the power $P$ for which the transmission band is narrow owing to our water-filling argument. Noting that the first derivative of $A(l, f) N(f)$ with respect to $f$ is zero at $f_{c}$; the Taylor series approximation has the form

$$
A(l, f) N(f) \approx A\left(l, f_{c}\right) N\left(f_{c}\right)+\Upsilon \frac{\left(f-f_{c}\right)^{2}}{2}
$$




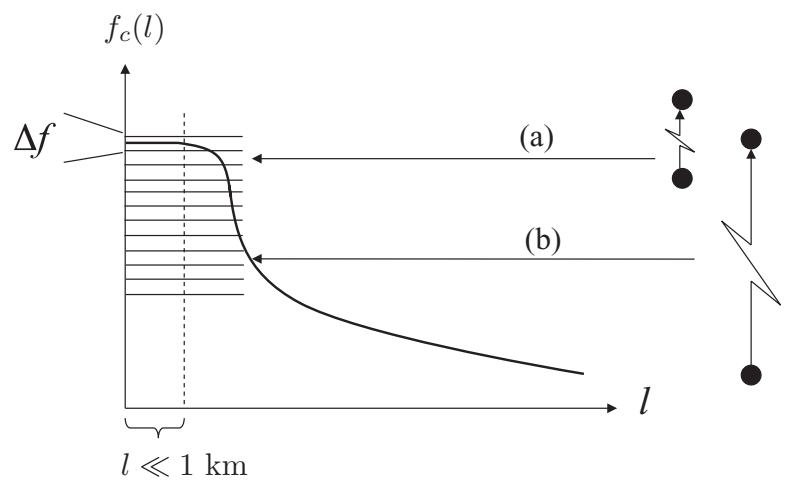

Figure 2. Relationship between transmission distance and center frequency in a narrow-band system.

$\forall f \in\left(f_{\min }, f_{\max }\right)$, where $\Upsilon=\left.\frac{\partial^{2}}{\partial f^{2}}(A(l, f) N(f))\right|_{f=f_{c}}$. Substituting this expression (2.5) into expression (2.4), and using the fact that

$$
K(l, C)=A\left(l, f_{\max }\right) N\left(f_{\max }\right)=A\left(l, f_{\min }\right) N\left(f_{\min }\right),
$$

where $f_{\max }$ and $f_{\min }$ are the maximum and minimum frequencies of the transmission band, we obtain

$$
P(l, C) \approx A\left(l, f_{\max }\right) N\left(f_{\max }\right) \Delta f-\int_{f_{\min }}^{f_{\max }}\left(A\left(l, f_{c}\right) N\left(f_{c}\right)+\Upsilon \frac{\left(f-f_{c}\right)^{2}}{2}\right) d f,
$$

where $\Delta f=f_{\max }-f_{\min }$. Considering $f_{\max }-f_{c} \approx \Delta f / 2$ and $f_{c}-f_{\min } \approx \Delta f / 2$, given our quadratic Taylor series approximation of $A(l, f) N(f)$, the above expression reduces to

$$
P=\frac{\Upsilon}{12} \Delta f^{3}
$$

\section{Fixed Narrow-Band Model}

We study the physical model of interference to obtain an upper bound on the transport capacity for transmission in an arbitrarily chosen narrow band in an underwater channel. The narrow-band assumption allows us to consider the attenuation as a constant over that band. Although we use similar assumptions in terms of node deployment and connection setup to those in [Gupta and Kumar 00], the steps to derive the upper bound change somewhat in order to accommodate a more complex path-loss model with different characteristics, 
e.g., $1 \leq \alpha \leq 2$ and $a(f) \geq 1$, instead of $\alpha \geq 2$ and $a(f)=1$ considered in [Gupta and Kumar 00] for radio channels. In fact, we show that the upper bound is expressed in terms of one of the branches of the Lambert function, which is an implicit function.

Remark 3.I. We limit our analysis to the far-field case, whereby receivers need to be at $r>\lambda /(2 \pi)$ for monopole or dipole sources [Bass et al. 03]. Note that for $c=1500 \mathrm{~m} / \mathrm{s}$ and a typical transmission frequency $f=10 \mathrm{kHz}$, we have

$$
r>\frac{c}{2 f \pi} \approx 0.024 \mathrm{~m}
$$

As an example, if we consider a grid topology deployed on an area of $1 \mathrm{~km}^{2}$, this means that the node density needs to be larger than $1.7 \cdot 10^{9}$ nodes $/ \mathrm{km}^{2}$ in order for the path loss to be dominated by near-field effects. From a practical perspective, nodes would have to be placed at distances in the order of a few centimeters from each other, while other physical constraints of underwater sensors, such as the dimensions of batteries, are typically larger than $0.024 \mathrm{~m}$. Such a large density of nodes is unlikely in real deployments, but makes scaling laws meaningful as an analysis technique well before reaching transmission distances dominated by near-field effects.

We assume that the nodes are arbitrarily deployed in a disk of unit area as in [Gupta and Kumar 00], that each node has an intended destination node, and that the requirement for successful reception at node $j$ of a transmission from node $i$ is

$$
\frac{P_{i}(f) / A\left(\left|X_{i}-X_{j(i)}\right|, f\right)}{N(f)+\sum_{k \in \tau, k \neq i} P_{k}(f) / A\left(\left|X_{k}-X_{j(i)}\right|, f\right)} \geq \beta,
$$

where $X_{i}$ is the position of node $i, X_{j(i)}$ is the position of node $j$ to which $i$ is transmitting, and $\tau$ is the set of all nodes transmitting simultaneously in the same transmission subband and time slot. We assume that all subbands are in the narrow band [Gupta and Kumar 00], so that the attenuation is dependent only on the central frequency of the narrow band. The above expression can also be written as

$$
\frac{P_{i}(f) / A\left(\left|X_{i}-X_{j(i)}\right|, f\right)}{N(f)+\sum_{k \in \tau} P_{k}(f) / A\left(\left|X_{k}-X_{j(i)}\right|, f\right)} \geq \frac{\beta}{\beta+1} .
$$

The parameter $f$ is maintained to emphasize the frequency dependence and to allow us to use these results in the following sections, where we analyze more complex settings. We consider that $\lambda$ is the throughput (bits/sec $=$ bps) of each node, that the network transports $\lambda n T$ bits over $T$ seconds, and that the 
average distance between source and destination of a bit is $\bar{L}$. As in [Gupta and Kumar 00], we define the transport capacity as $\lambda n \bar{L}$ bit-meters per second. Finally, we define $W=\triangle f \log _{2}(1+\beta)$ to be the transmission rate, where $\triangle f$ is the bandwidth of the narrow band chosen for transmission.

The following theorem summarizes the main result for the fixed narrow-band model.

Theorem 3.2. The transport capacity of an arbitrary underwater acoustic network under the fixed narrow-band model is bounded as follows:

$$
\lambda n \bar{L} \leq \Phi W n^{(\alpha-1) / \alpha} \exp \left(-W_{0}\left(\Phi \frac{2 \ln a(f)}{\alpha}\left(\frac{1}{n}\right)^{1 / \alpha}\right)\right),
$$

where

$$
\Phi=\frac{2^{1 / \alpha}}{\sqrt{\pi}}\left(\frac{\beta+1}{\beta}\right)^{1 / \alpha}\left(a(f)^{2 / \sqrt{\pi}}\right)^{1 / \alpha} .
$$

Proof. We define $r^{A}(l(h, b))=l(h, b)^{\alpha} a(f)^{l(h, b)}$, where $l(h, b)$ represents the distance between receiver and transmitter for the $h$ th hop of bit $b$, and $H$ is defined as the number of hops performed in $T$ seconds, which can be bounded by $H \leq W T n / 2$ [Gupta and Kumar 00].

Since $\left|X_{k}-X_{j(i)}\right| \leq 2 / \sqrt{\pi}$ for a disk of unit area, and $a(f) \geq 1, \forall f$, the path loss is

$$
A\left(\left|X_{k}-X_{j(i)}\right|, f\right) \leq\left(\frac{2}{\sqrt{\pi} l_{\mathrm{ref}}}\right)^{\alpha} a(f)^{2 / \sqrt{\pi}-l_{\mathrm{ref}}} \equiv \frac{\gamma_{\alpha}}{l_{\mathrm{ref}}^{\alpha} a(f)^{l_{\mathrm{ref}}}},
$$

where $\gamma_{\alpha}=(2 / \sqrt{\pi})^{\alpha} a(f)^{2 / \sqrt{\pi}}$. Using a similar procedure to that in [Gupta and Kumar 00], from (3.2), we have that

$$
A\left(\left|X_{i}-X_{j(i)}\right|, f\right) \leq \frac{\beta+1}{\beta} \frac{\gamma_{\alpha}}{l_{\mathrm{ref}}^{\alpha} a(f)^{l_{\mathrm{ref}}}} \frac{P_{i}(f)}{\sum_{k \in \tau} P_{k}(f)} .
$$

Let us sum over all transmitters $i \in \tau$ and use the definition of the path loss in expression (2.1):

$$
\sum_{i \in \tau}\left|X_{i}-X_{j(i)}\right|^{\alpha} a(f)^{\left|X_{i}-X_{j(i)}\right|} \leq \gamma_{\alpha} \frac{\beta+1}{\beta} .
$$

Summing over all subbands and time slots and dividing both sides by $H$, we obtain

$$
\frac{1}{H} \sum_{b=1}^{\lambda n T} \sum_{h=1}^{h(b)} r^{A}(h, b) \leq \gamma_{\alpha} \frac{\beta+1}{\beta} \frac{W T}{H}
$$


where $h(b)$ represents the $h$ th hop of a bit $b$. Since the function $r^{A}(l)=l^{\alpha} a(f)^{l}$ is increasing and convex for $l \geq 0, \alpha \geq 1$, and $a(f) \geq 1$, we have that

$$
r^{A}\left(\frac{1}{H} \sum_{b=1}^{\lambda n T} \sum_{h=1}^{h(b)} l(h, b)\right) \leq \gamma_{\alpha} \frac{\beta+1}{\beta} \frac{W T}{H} .
$$

Let us define $\psi=(\ln a(f))^{\alpha} \gamma_{\alpha} \frac{\beta+1}{\beta} \frac{W T}{H}$, and note that $\psi \geq 0$. Noticing that the left-hand side of the above inequality is a Lambert function of the form $W^{\alpha} \exp W$, which is an increasing function when $W \geq 0$, is one of the key steps in our proof that are different from the work in [Gupta and Kumar 00]. Hence,

$$
\frac{\ln a(f)}{H} \sum_{b=1}^{\lambda n T} \sum_{h=1}^{h(b)} l(h, b) \leq \psi^{1 / \alpha} \exp \left(-W_{0}\left(\frac{\psi^{1 / \alpha}}{\alpha}\right)\right)
$$

where $W_{0}(\cdot)$ is the branch zero of the Lambert function, using the nomenclature of [Chapeau-Blondeau and Monir 02]. This fact implies that

$$
\lambda n \bar{L} \leq \frac{H}{T \ln a(f)} \psi^{1 / \alpha} \exp \left(-W_{0}\left(\frac{\psi^{1 / \alpha}}{\alpha}\right)\right) .
$$

Substituting for $\psi$ in (3.10), we obtain

$$
\lambda n \bar{L} \leq \frac{H^{(\alpha-1) / \alpha}}{T^{(\alpha-1) / \alpha}}\left(\gamma_{\alpha} \frac{\beta+1}{\beta} W\right)^{1 / \alpha} \exp \left(-W_{0}\left(\frac{\psi^{1 / \alpha}}{\alpha}\right)\right) .
$$

Since $H^{(\alpha-1) / \alpha}$ is an increasing function for $\alpha>1$, and constant for $\alpha=1$, it follows that $H^{(\alpha-1) / \alpha} \leq\left(\frac{W T n}{2}\right)^{(\alpha-1) / \alpha}$. Another important step of our proof, different from [Gupta and Kumar 00], is to note that $W_{0}(\cdot)$ is an increasing function. Hence, we have that

$$
W_{0}\left(\frac{\psi^{1 / \alpha}}{\alpha}\right) \geq W_{0}\left(\frac{2 \ln a(f) a(f)^{2 / \alpha \sqrt{\pi}}}{\alpha \sqrt{\pi}}\left(\frac{\beta+1}{\beta}\right)^{1 / \alpha} \frac{2^{1 / \alpha}}{n^{1 / \alpha}}\right) .
$$

Substituting these inequalities into expression (3.11) concludes the proof.

Since the branch zero of the Lambert function satisfies $W_{0}(x) \geq 0, \forall x \geq 0$, the exponential term $\exp \left(-W_{0}\left(O\left(n^{-1 / \alpha}\right)\right)\right)$ has values between 0 and 1 . Note that as $n \rightarrow \infty$, the exponential term in the scaling law goes to 1 . This implies that the exponential term influences the scaling for small $n$, while for large enough $n$, the upper bound is $O\left(n^{(\alpha-1) / \alpha}\right)$. However, the exponential term will be instrumental for Section 6 , where we consider a transmission frequency that scales with respect to the network size. 
Remark 3.3. If we consider $a(f)=1$, i.e., the same path-loss model as in [Gupta and Kumar 00], and recall that $W_{0}(0)=0$, we have

$$
\lambda n \bar{L} \leq \frac{1}{\sqrt{\pi}}\left(\frac{2 \beta+2}{\beta}\right)^{1 / \alpha} W n^{(\alpha-1) / \alpha},
$$

which is the original result of [Gupta and Kumar 00]. We have thus proved that the result in [Gupta and Kumar 00] is valid for $\alpha \geq 1$.

\section{I. Numerical Results}

Figures 3 and 4 illustrate the upper bound on $\lambda \bar{L}$ for different values of $a(f)$ ranging from 1 to 10000 , which are characteristic of an underwater environment

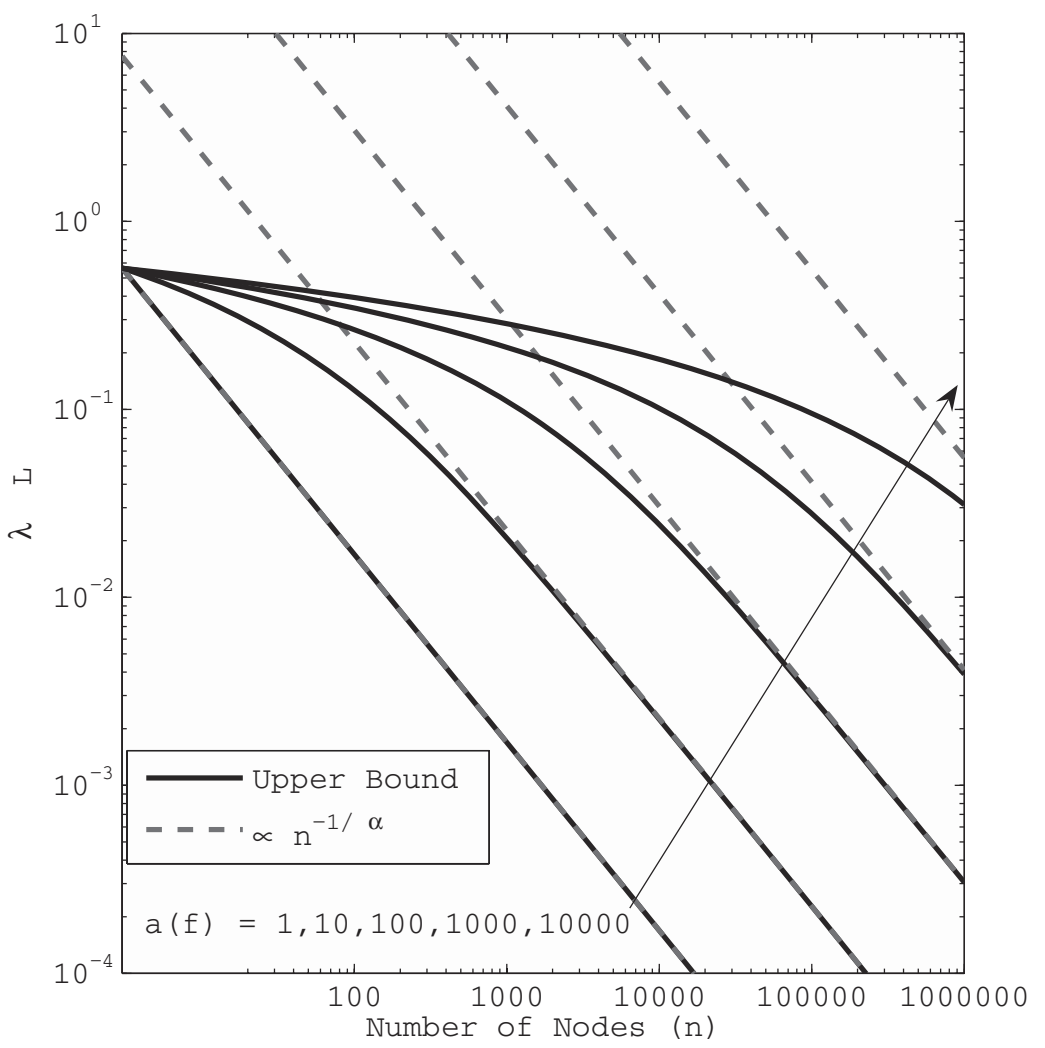

Figure 3. Upper bound on $\lambda \bar{L}$ for an arbitrarily chosen narrow band and different values of $a(f): W=1 \mathrm{bps}, \alpha=1, \beta=2$, area $=1 \mathrm{~km}^{2}$. 


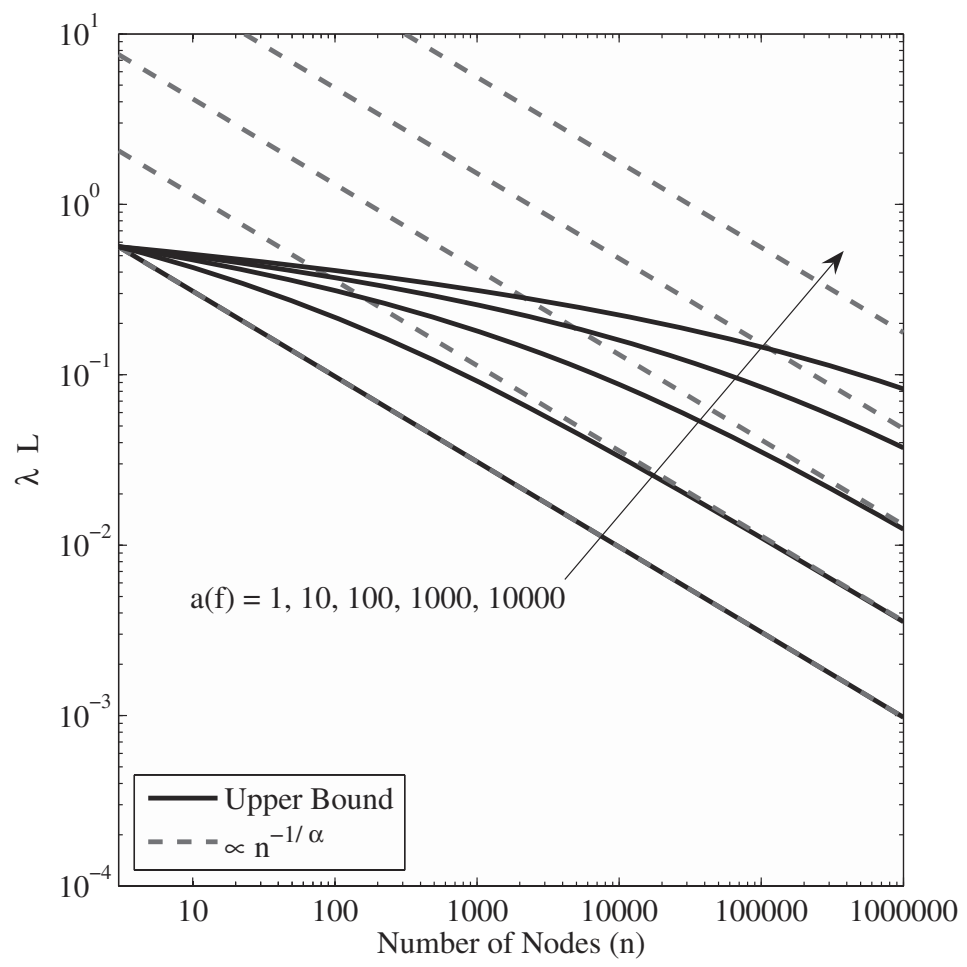

Figure 4. Upper bound on $\lambda \bar{L}$ for an arbitrarily chosen narrow band and different values of $a(f): W=1 \mathrm{bps}, \alpha=2, \beta=2$, area $=1 \mathrm{~km}^{2}$.

at different frequencies with $l$ in kilometers. For example, $a(f)=1000$ corresponds to a frequency of around $100 \mathrm{kHz}$. We have used $\alpha=1$ and $\alpha=2$ and the parameters specified in Figures 3 and 4, respectively. We also plot dashed lines proportional to $n^{-1 / \alpha}$. As expected, as $n$ becomes larger, the exponential term of the upper bound becomes negligible, making the bound scale grow as $O\left(n^{-1 / \alpha}\right)$. However, for small values of $n$, the bound begins at a common point for the different $a(f)$ values and decays very slowly.

Figures 3 and 4 also illustrate that the value of $a(f)$ determines the transition between these two operating regions: the larger $a(f)$ is, the greater $n$ has to be before transitioning. Of course, if we use a transmission band with high $a(f)$, each node will have to be able to transmit at higher power to reach its destination. In the underwater channel, this also means that a higher center frequency is required, because $a(f)$ is an increasing function of $f$.

Figures 3 and 4 show that $\lambda \bar{L}$ remains almost constant for $n \leq 100$ nodes, $a(f)>100$, and a disk area of $1 \mathrm{~km}^{2}$, which corresponds to densities of up to 
100 nodes per $\mathrm{km}^{2}$. The expected density of nodes in an underwater network is usually much lower given the current applications for which they are deployed, e.g., environmental measurements. Thus, the bound on $\lambda \bar{L}$ is almost constant for this simple case. Similar results will apply in most practical scenarios with appropriate modifications to account for specific deployments. Of course, actual performance will depend on the protocols used in practice.

Remark 3.4. From Figures 3 and 4, it is clear that there are two main regions of operation, the transition depending on $a(f), n$, and the area of deployment. The schemes in Section 3.2 provide specific cases that follow this two-region behavior. In a sense, the two regions constitute a power-limited region and an interference-limited region. This dependence implies that the application of our result to deployments in which we expand the deployment area requires a bit more care than for the case of [Gupta and Kumar 00], because expanding the area will influence the transition point among the two regions. However, it does not compromise the impact of our results.

\subsection{Constructive Scheme}

Aiming to understand these fundamental limits, we propose a constructive scheme to illustrate that (i) a transport capacity scales as $o(\sqrt{n})$ for underwater channels when we rely on a fixed $a(f)$, i.e., fixed transmission frequency, and (ii) there exist topologies and schemes that follow the two-region scaling observed in Section 3.1.

Scheme 3.5. Let us consider the following placement of nodes in a square contained in a disk of area 1 . We consider that transmitters and receivers are located at the following $(x, y)$ coordinates:

$$
(j(4 d) \pm d, k(4 d))
$$

and

$$
(j(4 d), k(4 d) \pm d),
$$

where the case of $|j+k|$ odd corresponds to the transmitters, and $|j+k|$ even corresponds to the receivers. We consider that each transmitter sends information to its closest receiver and assume that $d \propto \min \left(d_{0}, 1 /(4 \sqrt{n})\right)$.

Scheme 3.5 provides placements that are at a fixed distance $d_{0}$, while the placement of the nodes does not exceed the boundaries of the region of deployment. After that, the distance between nodes scale as $O(1 / \sqrt{n})$. Lemma 3.6 provides bounds on the interference, which are later used to prove other key results. 
Lemma 3.6. The interference $I$ of a receiver in Scheme 3.5 for the case $d=$ $O(1 / \sqrt{n})$ is bounded as

$$
I \leq \mathcal{C} \sum_{l=1}^{\sqrt{n} / 8} \frac{(l+1) P}{(3 d l)^{\alpha} a(f)^{3 l d}}
$$

where $\mathcal{C}$ is a constant, and

$$
I \geq \mathcal{C}^{\prime} \sum_{l=1}^{\sqrt{n} / 8} \frac{P}{(5 d l)^{\alpha} a(f)^{5 l d}}
$$

where $\mathcal{C}^{\prime}$ is a constant

Proof. We can bound the interference of Scheme 3.5 from above by considering one of the receivers at $|j+k|=0$, which has a signal coming from its closest transmitter at distance $2 d$. The interference is lower than if we considered that the interference from every transmitter in a neighborhood located at $|j|=l$ and $|k|=l$, with $l>0$ and integer-valued, came from a transmission at a distance $4 l d-d$. This is further bounded if we consider that all nodes in a neighborhood with $|j|=l$ and $|k|=l$ are transmitters, i.e., we will have $4 l+4$ transmitters for a given $l$. After some manipulations, we obtain the upper bound.

The lower bound follows by considering one of the receivers at $|j+k|=0$, which has a signal coming from its closest transmitter at distance $2 d$. The interference is higher than if we considered that the interference from every transmitter in a neighborhood located at $|j|=l$ and $|k|=l$, with $l>0$ and integer-valued, came from a transmission at a distance $5 l d$. This is further bounded if we consider that all nodes in a neighborhood with $|j|=l$ and $|k|=l$ and $l>0$ are not transmitting, except for those placed in $|j|=l$ and $k=0$. Finally, we consider that we will have three transmitters for a given $l$.

Theorem 3.7. The signal to interference plus noise ratio (SINR) for receivers in Scheme 3.5 is bounded as

$$
\begin{aligned}
& \mathrm{SINR} \geq \frac{1}{\mathcal{C} \sum_{l=1}^{\sqrt{n} / 8}\left(l^{1-\alpha}+l^{-\alpha}\right) a(f)^{-3 l d+2 d}+N(f) a(f)^{2 d}(2 d)^{\alpha} / P}, \\
& \mathrm{SINR} \leq \frac{1}{\mathcal{C}^{\prime} \sum_{l=1}^{\sqrt{n} / 8} l^{-\alpha} a(f)^{-5 l d+2 d}+N(f) a(f)^{2 d}(2 d)^{\alpha} / P},
\end{aligned}
$$

for constants $\mathcal{C}$ and $\mathcal{C}^{\prime}$ 
Proof. The proof follows from considering

$$
\mathrm{SINR}=\frac{P /\left((2 d)^{\alpha} a(f)^{2 d}\right)}{I+N(f)}
$$

and using the bounds of Lemma 3.6.

Let us consider the case $\alpha=1$ as an example of the presence of two main operation regions related to characteristics of the channel. The first region is related to small values of $n$ where the transport capacity increases at least as fast as $O(\sqrt{n})$. For larger $n$, a transport capacity of $O(\sqrt{n})$ cannot be supported, because the SINR strictly decreases as $n$ increases.

Theorem 3.8. Scheme 3.5 for $\alpha=1$ supports a transport capacity of

$$
\begin{aligned}
& \lambda n \bar{L}=O(n) \quad \text { for } n \leq \frac{1}{4 d_{0}^{2}}, \\
& \lambda n \bar{L}=O(\sqrt{n}) \quad \text { for } n \leq \frac{3 \ln a(f)}{\ln (1 / \epsilon)} \text { if } \frac{3 \ln a(f)}{\ln (1 / \epsilon)}>\frac{1}{4 d_{0}^{2}}, \\
& \lambda n \bar{L}=o(\sqrt{n}) \text { for } n>\max \left(\frac{1}{4 d_{0}^{2}}, \frac{3 \ln a(f)}{\ln (1 / \epsilon)}\right),
\end{aligned}
$$

for $\epsilon \in(0,1)$.

Proof. The proof of the first two parts of the theorem follows from considering the lower bound on the SINR from Theorem 3.7. The last part of the theorem follows from considering the upper bound on the SINR. If we consider $n \leq 1 / 4 d_{0}^{2}$, then $d \propto d_{0}$, which means that $\bar{L}=O(1)$. For this region, $N(f) a(f)^{2 d_{0}}\left(2 d_{0}\right)^{\alpha} / P$ is a constant; $\sum_{l=1}^{\sqrt{n} / 8} l^{-\alpha} a(f)^{-3 l d_{0}+2 d_{0}}$ is bounded above by $\sum_{l=1}^{\infty} l^{-\alpha} a(f)^{-3 l d_{0}+2 d_{0}}$, which is a constant for $\alpha \geq 1$, and $\sum_{l=1}^{\sqrt{n} / 8} l^{1-\alpha} a(f)^{-3 l d_{0}+2 d_{0}}$ for $\alpha \geq 1$. Thus, a minimum SINR value $\beta$ can be maintained, and $\lambda n \bar{L}=O(1)$ for $n \leq 1 / 4 d_{0}^{2}$.

If

$$
\frac{1}{4 d_{0}^{2}}<n \leq \frac{3 \ln a(f)}{\ln (1 / \epsilon)},
$$

then $d=O(1 / \sqrt{n})$, which means that $\bar{L}=O(1 / \sqrt{n})$. For this region and $\alpha=1$, we have

$$
\sum_{l=1}^{\sqrt{n} / 8} l^{-1} a(f)^{-3 l d+2 d}=a(f)^{2 d} \sum_{l=1}^{\sqrt{n} / 8} l^{-1} a(f)^{-3 l d} \leq-a(f)^{2 d} \ln \left(1-a(f)^{-3 d}\right) .
$$

Thus, for the defined region, we have $-\ln \left(1-a(f)^{-3 d}\right)<-\ln (1-\epsilon)$. Since $N(f) a(f)^{2 d}(2 d)^{\alpha} / P$ decreases to zero as $n$ increases with $d=O(1 / \sqrt{n})$, it follows that a given SINR value can be supported, and thus $\lambda n \bar{L}=O(1 / \sqrt{n})$. 
If

$$
n>\max \left(\frac{1}{4 d_{0}^{2}}, \frac{3 \ln a(f)}{\ln (1 / \epsilon)}\right),
$$

we use the upper bound on the SINR to prove that the SINR strictly decreases as $n \rightarrow \infty$. Through simple manipulations, we prove that since $d=$ $O(1 / \sqrt{n})$, it follows that $N(f) a(f)^{2 d}(2 d)^{\alpha} / P \rightarrow 0$. We now focus on the term $a(f)^{2 d} \sum_{l=1}^{\sqrt{n} / 8} l^{-1} a(f)^{-5 l d}$, where

$$
\sum_{l=1}^{\sqrt{n} / 8} l^{-1} a(f)^{-5 l d} \geq a(f)^{2 d} a(f)^{-(5 / 8) \sqrt{n} d} \sum_{l=1}^{\sqrt{n} / 8} l^{-1} .
$$

Since for large $n$, we have $d=O(1 / \sqrt{n})$, the term $a(f)^{2 d} a(f)^{-(5 / 8) \sqrt{n} d}$ is a constant and $\sum_{l=1}^{\sqrt{n} / 8} l^{-1}$ is a divergent series. This implies that $\lambda n \bar{L}=O(\sqrt{n})$ is not sustainable, and thus $\lambda n \bar{L}=o(\sqrt{n})$, concluding the proof.

\section{Transmissions over a Wide Band}

This section considers two cases in which a wide band is available: the case of low power, whereby multiple narrow-band transmissions are performed, and the case of high power, in which the wide band is used by all transmitters. We extend the previous upper bounds for these scenarios.

\section{I. Low-Power-Narrow-Band Case}

As mentioned in the introduction, one of the characteristics of the underwater acoustic channel is that the optimal transmission band using the water-filling principle depends strongly on the distance of a link [Stojanovic 07]. In particular, if the transmission power of a node is very low, then nodes will optimally transmit in different bands corresponding to different transmission distances. This was shown in (2.6), which provides a measure of the required transmission power under Gaussian noise. Thus, interference will come only from nodes transmitting in the same band. We derived an expression for the power under these assumptions in Section 2. In order to assign disjoint transmission bands, we divide the total transmission band of the system into nonoverlapping bands of width $\Delta f$. We use $f_{c}(l)$ as the mapping between the transmission distance and the corresponding transmission band for a low-power-narrow-band scenario. Thus, if a node transmits to another node at a distance $l$, we assign the transmission band centered at the frequency $f_{c}(l)$ as in Figure 2 . 
The capacity analysis is similar to that in Section III if $a(f)$ is replaced by $a\left(f_{m}\right)$ for each of the bands, where $f_{m}$ is the central frequency of transmission band $m$. Note that this analysis is inherently different from that in [Gupta and Kumar 00], which does not consider any frequency dependence of the path-loss model. Let us assume that each node is capable of transmitting at $\triangle W$ bps in each band, where $\triangle W=\triangle f \log _{2}(1+\beta)$, and $\triangle f$ is the bandwidth of each nonoverlapping band.

The definition of $H$ changes slightly when we allow multinode hopping for the low-power-narrow-band case in order to incorporate the effect of the different transmission bands. In this case,

$$
H \leq \frac{T|\Gamma| \triangle W n}{2}=\frac{T W n}{2},
$$

where $\Gamma$ is the set of subbands used by the network, and $W=|\Gamma| \triangle W$. At this point, we define $\gamma_{\alpha}\left(f_{m}\right)$ as $\gamma_{\alpha}$ for band $m$. We use the fact that $a\left(f_{m}\right) \geq a_{\min }$, where $a_{\min }=\min _{m \in \Gamma} a\left(f_{m}\right)$. In the underwater scenario, $a_{\min }=a\left(f_{\min }\right)$, because $a(f)$ is an increasing function of $f$.

Theorem 4.I. The transport capacity for arbitrary underwater acoustic networks under the low-power-narrow-band case is bounded by

$$
\lambda n \bar{L} \leq \Phi W n^{(\alpha-1) / \alpha} \exp \left(-W_{0}\left(\Phi \frac{2 \ln a\left(f_{\min }\right)}{\alpha}\left(\frac{1}{n}\right)^{1 / \alpha}\right)\right),
$$

where

$$
\Phi=\frac{2^{1 / \alpha}}{\sqrt{\pi}}\left(\frac{\beta+1}{\beta}\right)^{1 / \alpha}\left(\frac{1}{|\Gamma|} \sum_{m \in \Gamma} a\left(f_{m}\right)^{2 / \sqrt{\pi}}\right)^{1 / \alpha}
$$

Proof. For each of the different bands, the analysis is as before up to (3.6). Summing over all subbands and time slots, we obtain

$$
\sum_{s \in S} \sum_{m \in \Gamma} \sum_{i \in \tau}\left|X_{i}-X_{j(i)}\right|^{\alpha} a\left(f_{m}\right)^{\left|X_{i}-X_{j(i)}\right|} \leq \frac{\beta+1}{\beta} \triangle W T \sum_{m \in \Gamma} \gamma_{\alpha}\left(f_{m}\right),
$$

where $S$ is the set of time slots. Defining $r^{A}\left(h, b, f_{\min }\right)=l(h, b)^{\alpha} a\left(f_{\min }\right)^{l(h, b)}$ and following similar steps to those leading to (3.7), we get

$$
\frac{1}{H} \sum_{b=1}^{\lambda n T} \sum_{h=1}^{h(b)} r^{A}\left(h, b, f_{\min }\right) \leq \frac{\beta+1}{\beta} \frac{\triangle W T}{H} \sum_{m \in \Gamma} \gamma_{\alpha}\left(f_{m}\right) .
$$


Defining

$$
\psi=\left(\ln a\left(f_{\min }\right)\right)^{\alpha} \frac{\beta+1}{\beta} \frac{\triangle W T}{H} \sum_{m \in \Gamma} \gamma_{\alpha}\left(f_{m}\right),
$$

we can use a similar procedure to that in the previous section to show that

$$
\lambda n \bar{L} \leq \frac{H^{(\alpha-1) / \alpha}}{T^{(\alpha-1) / \alpha}}\left(\frac{\beta+1}{\beta} W \sum_{m \in \Gamma} \gamma_{\alpha}\left(f_{m}\right)\right)^{1 / \alpha} \exp \left(-W_{0}\left(\frac{\psi^{1 / \alpha}}{\alpha}\right)\right)
$$

Using the inequality $H \leq T W n / 2$, we obtain the upper bound on transport capacity. This concludes the proof.

The scaling law is similar in structure to the one obtained in Section 3. However, the constant $\Phi$ depends on the average of a function of the absorption coefficients at $f_{m} \forall m \in \Gamma$, instead of a particular value. Again, if $a(f)=1 \forall f$, the result reduces to that of [Gupta and Kumar 00].

Remark 4.2. (Direct transmissions.) If we constrain our system to perform direct transmissions only (single hop), using the fact that there is an assignment of frequency bands in terms of the distance, we can consider that $h(b)=1 \forall b$, i.e., only one hop. Given the distance-band separation property mentioned in previous sections, the problem can be thought of as solving for several networks that lie on top of each other, in different layers with no cross-layer interference. Membership in the layers is based on the distance of the connection. In other words, each transmission band $m$ will have $n_{m}$ transmitters, where $n=\sum_{m \in \Gamma} n_{m}$ constitutes the total number of nodes in the network, since each transmitter has only one intended destination.

These facts cause a different capacity scaling for each of the transmission bands, i.e., the scaling for each transmission band will have the form of (3.3) with $\triangle W$ instead of $W$ and $n_{m}$ instead of $n$ to obtain the scaling for band $m$.

\subsection{High-Power-Wide-Band Case}

In the high-power-wide-band scenario, nodes have enough power to transmit in a wide band $B$, which implies that the absorption cannot be considered to be constant over the band. The band $B$ is again chosen using a water-filling argument. We shall use two different SINR requirements. The first is meant for OFDM-like (orthogonal frequency-division multiplexing) schemes, whereby each transmission band may need a specific SINR to guarantee transmission. We then specify the changes that arise in using a more standard SINR definition for wide-band transmissions. 
Let us first consider the case of an SINR requirement that depends on the frequency, that is,

$$
\frac{P_{i}(f) / A\left(\left|X_{i}-X_{j(i)}\right|, f\right)}{N(f)+\sum_{k \in \tau, k \neq i} P_{k}(f) / A\left(\left|X_{k}-X_{j(i)}\right|, f\right)} \geq \beta(f) .
$$

Remark 4.3. This SINR requirement is relevant for an idealized OFDM scheme over the fixed transmission band $B$, where the system uses transmission channels of infinitesimal width and no guard band between channels.

We define $f_{\min }=\arg \min _{f} a(f)$, while $H$ can be shown to have the bound $H \leq T W n / 2$ using the definition of $W$; see (4.3). We define $W$ as the data rate over the entire band, computed as

$$
W=\int_{f \in B} \log _{2}(1+\beta(f)) d f .
$$

If we assign a transmission rate to every subband $d f$ of $d W=\log _{2}(1+\beta(f)) d f$, the analysis for each frequency is similar to that in Section 4 . Letting $\triangle f \rightarrow 0$, renaming $\triangle f$ as $d f$, and replacing the sums by integrals, we have obtained the following result.

Theorem 4.4. The transport capacity of an arbitrary underwater acoustic network under the high-power-wide-band case under the SINR condition (4.2) is bounded as follows:

$$
\lambda n \bar{L} \leq \Theta W n^{(\alpha-1) / \alpha} \exp \left(-W_{0}\left(\Theta \frac{2 \ln a\left(f_{\min }\right)}{\alpha}\left(\frac{1}{n}\right)^{1 / \alpha}\right)\right),
$$

where

$$
\Theta=\frac{2^{1 / \alpha}}{\sqrt{\pi}}\left(\frac{1}{W} \int_{B} \frac{(\beta(f)+1) a(f)^{2 / \sqrt{\pi}} \log _{2}(1+\beta(f))}{\beta(f)} d f\right)^{1 / \alpha}
$$

Proof. Using similar techniques to those in previous sections, we obtain

$$
\begin{aligned}
\frac{1}{H} \sum_{b=1}^{\lambda n T} \sum_{h=1}^{h(b)} W r^{A}\left(h, b, f_{\text {min }}\right) & \leq \frac{T}{H} \int_{W} \frac{\beta(f)+1}{\beta(f)} \gamma_{\alpha}(f) d W \\
& =\frac{T}{H} \int_{B} \frac{\beta(f)+1}{\beta(f)} \gamma_{\alpha}(f) \log _{2}(1+\beta(f)) d f .
\end{aligned}
$$

Following the procedure of Section 4 concludes the proof. 
Remark 4.5. We now consider the alternative case of an SINR requirement for the full transmission band, that is,

$$
\frac{\int_{B} P_{i}(f) / A\left(\left|X_{i}-X_{j(i)}\right|, f\right) d f}{\int_{B} N(f)+\sum_{k \in \tau, k \neq i} P_{k}(f) / A\left(\left|X_{k}-X_{j(i)}\right|, f\right) d f} \geq \beta .
$$

The scaling result is straightforward from the proof of Theorem 3.2, where the key step lies in using the inequality

$$
\int_{B} \frac{P_{i}(f)}{A\left(\left|X_{i}-X_{j(i)}\right|, f\right)} d f \leq \frac{\int_{B} P_{i}(f) d f}{A\left(\left|X_{i}-X_{j(i)}\right|, f_{\min }\right)} .
$$

The scaling is the same as for Theorem 3.2 , with $a(f)$ substituted by $a\left(f_{\min }\right)$.

\section{Scaling Transmission Frequency with the Number of Nodes}

We have observed that the transport capacity scales much more slowly than the capacity of a simple multihop scheme in radio wireless, i.e., much more slowly than $O\left(n^{1 / 2}\right)$. This section shows that scaling the transmission frequency with the number of nodes provides a means to overcome this limitation. We first study the structure of the upper bound and then show a simple scheme that achieves a transport capacity of at least $O\left(n^{1 / 2}\right)$, regardless of the parameter $\alpha$ that limited our previous results. We assume the case of a fixed narrow band for simplicity, although this can be extended to more complex cases.

\section{I. An Upper Bound to Transport Capacity}

We show in the following that the transport capacity can have an upper bound that scales as $O\left(n^{1-\epsilon}\right)$ for an arbitrary $\epsilon>0$. We prove this by assuming that such an upper bound is possible and showing the corresponding scaling of $f$ that satisfies it. We use the notation $f(n)$ to emphasize the dependence on the number of nodes in the network. We consider the scaling of the absorption $a(f(n))$ with the number of nodes for simplicity. As explained in Section 2, $a(f)$ is an increasing function on $f$ for the frequency range of interest, so there is a one-to-one mapping between the values of $a(f)$ and $f$. We exploit this fact in order to derive our new transport capacity upper bound. This result is summarized in the following theorem.

Theorem 5.I. The transport capacity $\lambda n \bar{L}$ of underwater acoustic networks with a fixed narrow-band assumption and the transmission frequency $f(n)$ scaling with 
the number of nodes is bounded by

$$
\begin{aligned}
& O\left(n^{(\alpha-1) / \alpha} a(f(n))^{2 / \sqrt{\pi} \alpha} \exp \left(-W_{0}\left(n^{-1 / \alpha} a(f(n))^{2 / \sqrt{\pi} \alpha}\right)\right)\right) \\
& \quad \text { if } f(n)=o(\sqrt{\ln (n)}), \\
& O\left(n^{1-\epsilon}\right) \text { if } f(n)=O(\sqrt{\ln (n)}), \text { with } \epsilon>0, \\
& O(n) \quad \text { if } f(n)=\Omega(\sqrt{\ln (n)}) .
\end{aligned}
$$

Proof. The first case is a straightforward operation considering the result in Theorem 3.2. We shall focus first on proving the case $f(n)=O(\sqrt{\ln (n)})$. Considering expression (3.3), note that to ensure that the transport capacity will be bounded by $O\left(n^{1-\epsilon}\right)$, we require

$$
a(f(n))^{2 / \sqrt{\pi} \alpha}=n^{1 / \alpha-\epsilon},
$$

without considering the effect of the exponential term. However, it is simple to check that the limit of the exponential term when $n \rightarrow \infty$ is given by

$$
\lim _{n \rightarrow \infty} \exp \left(-W_{0}\left(C(\alpha, \epsilon, \beta) n^{-\epsilon} \ln (n)\right)\right)=1,
$$

where $C(\alpha, \epsilon, \beta)$ is a constant that depends on $\alpha, \epsilon$, and $\beta$.

Considering expression (2.2), it straightforward to determine that the nodedependent frequency $f(n)$ scales on the number of nodes as

$$
f(n)=O(\sqrt{\ln (n)}) .
$$

We conclude that for $f(n)=O(\sqrt{\ln (n)})$, the transport capacity is bounded as

$$
\lambda n \bar{L} \leq D(\alpha, \epsilon, \beta, W) n^{1-\epsilon} \exp \left(-W_{0}\left(C(\alpha, \epsilon, \beta) n^{-\epsilon} \ln (n)\right)\right),
$$

where $D(\alpha, \epsilon, \beta, W)$ is a constant that depends on $\alpha, \epsilon, \beta$, and $W$. For large $n$, the bound becomes

$$
\lambda n \bar{L} \leq D(\alpha, \epsilon, \beta, W) n^{1-\epsilon} .
$$

We follow a similar technique to prove the remaining case.

This result is very important, not only because it relaxes our upper bound on the transport capacity, but because it can be achieved with a scaling on the frequency that is fairly slow, i.e., $f(n)=O(\sqrt{\ln (n)})$. This makes our result useful for a wide range of $n$ while mapping it to relevant transmission frequencies that are feasible in practice. 


\subsection{Constructive Lower Bound}

Let us now show a placement of nodes and assignment of traffic patterns that overcomes the initial limitation on the transport capacity for any value of $\alpha$, achieving a scaling of $O\left(n^{1 / 2}\right)$. In the cases of a fixed transmission band, we showed an upper bound that stated that for $1 \leq \alpha<2$, this was not possible. The result is summarized in the following theorem.

Theorem 5.2. There is a placement of nodes and an assignment of traffic patterns such that the transport capacity achieves $\lambda n \bar{L}=O(\sqrt{n})$ for $f(n)=O\left(n^{1 / 4}\right)$ for underwater acoustic networks.

Proof. Using Scheme 3.5 and the lower bound in Theorem 3.7, we will confirm that we can provide an SINR level such that $\operatorname{SINR} \geq \beta$.

First, we observe that the term $\mathcal{C}^{\prime} \sum_{l=1}^{\sqrt{n} / 8}\left(l^{1-\alpha}+l^{-\alpha}\right) a(f(n))^{-3 l d+2 d}$ is bounded as long as $a(f(n))^{-3 d}=o(1)$ or $a(f(n))^{-3 d}=O(1)$. One instance that satisfies this condition occurs if (1) we use the scaling of $a(f)=O\left(e^{\sqrt{n}}\right)$, which is associated with $f(n)=O\left(n^{1 / 4}\right)$, and (2) we also use the fact that $d=O(1 / \sqrt{n})$. This implies that as $n \rightarrow \infty, a(f(n))^{-3 d} \rightarrow e^{-c_{1}}<1$, where $c_{1}>0$.

Second, since the overall psd of the noise $N(f)$ decays linearly on the logarithmic scale in the frequency region from $100 \mathrm{~Hz}$ to $100 \mathrm{kHz}$, which is the operating regime used by the majority of acoustic systems and is approximately given by $\log N(f)=a_{4}-a_{5} \log f$ for some positive constants $a_{4}$ and $a_{5}>0$ independent of $n$ [Stojanovic 07], we can assume the scaling of $N(f)$ to be given by $N(f)=O\left(f^{-a_{5}}\right)$. This implies that the term $N(f) a(f)^{2 d}(2 d)^{\alpha} / P$ approaches zero as $n \rightarrow \infty$ if we assume also $a(f)=O\left(e^{\sqrt{n}}\right)$ and $d=O(1 / \sqrt{n})$.

Thus, we will be able to satisfy any condition SINR $\geq \beta$ with sufficiently large $n$. Finally, since the average distance $\bar{L}$ between transmissions grows as $O\left(n^{-1 / 2}\right)$, we conclude that the transport capacity for this placement scales as $O(\sqrt{n})$, regardless of the value of $\alpha$.

Remark 5.3. As shown in the proof of Theorem 5.2, the placement specified allows for the SINR to be as large as required for sufficiently large $n$ for the receivers to successfully recover the data packets. This is unique with respect to the result of [Gupta and Kumar 00] (i.e., $a(f)=1$ and $\alpha \geq 2$ ), where the SINR is bounded but converges to a specific value as $n \rightarrow \infty$. This implies that the lower bound $O(\sqrt{n})$ for the transport capacity may not be tight and that other placements may achieve a better scaling as predicted by Theorem 5.1. This will constitute the focus of our future work. 


\section{Conclusions}

This work presents upper bounds on the transport capacity of underwater acoustic networks with nodes deployed arbitrarily in a unit-area disk. We study three cases of interest: an arbitrarily chosen narrow transmission band, the case of power-limited nodes that transmit in disjoint narrow bands, and the case of nodes with high-power capabilities that use a wide transmission band. The choice of transmission band in the last two cases depends on the transmission distance and the physical characteristics of the channel and is made in accordance with the water-filling principle. We also study the case in which the transmission frequency scales with the number of nodes and provide upper and lower bounds for this case.

We have shown that the amount of information that can be exchanged by each source-destination pair in an underwater acoustic network goes to zero as the number of nodes $n$ goes to infinity, at least at a rate $n^{-1 / \alpha} e^{-W_{0}\left(O\left(n^{-1 / \alpha}\right)\right)}$. This rule is valid for the different scenarios in general, requiring only changes in the scaling constants. The throughput per source-destination pair has two different regions. For small $n$, the throughput decreases very slowly as $n$ increases. For large $n$, it decreases as $n^{-1 / \alpha}$. Given that $1 \leq \alpha \leq 2$ in an underwater acoustic channel, the available throughput for large $n$ decays more rapidly than in typical radio wireless networks. However, typical node densities in underwater networks correspond to the small- $n$ regime. In a narrow-band example with values of $a(f)$ characteristic of an underwater channel, we showed that the upper bound on the throughput remains almost constant for densities up to 100 nodes per $\mathrm{km}^{2}$. Most underwater networks have node densities in this range owing to the applications for which they are deployed.

We have also shown that scaling the frequency with the number of nodes provides a means of overcoming the limitations in transport capacity for underwater acoustic networks. This is especially interesting if the number of nodes becomes large. We presented a scheme that could achieve a transport capacity scaling of $O(\sqrt{n})$, which was not possible in the fixed-transmission-band cases for values of $\alpha \in[1,2)$, with $\alpha=2$ being the only exception.

Finally, we have identified some important characteristics of the underwater acoustic channel useful for future studies. For example, we could allow cooperation between nodes à la [Ozgur et al. 07], taking advantage of the distance-band separation property of the underwater channel. Namely, instead of performing time division between long and short transmissions, we could simply transmit in different bands that do not interfere with one another. This is important because acoustic transmissions have long propagation delays due to the speed of 
sound underwater $(\approx 1500 \mathrm{~m} / \mathrm{s})$, which reduces the usefulness of a time-division scheme. Another interesting area is the study of different operational regions in which we use different scaling functions for the frequency given the number of nodes $n$.

Acknowledgments. This work was supported in part by the National Science Foundation under grants No. 0520075, 0831728, and CNS-0627021, by ONR MURI Grant No. N00014-07-1-0738, and subcontract \# 060786 issued by BAE Systems National Security Solutions, Inc., and supported by the Defense Advanced Research Projects Agency (DARPA) and the Space and Naval Warfare System Center (SPAWARSYSCEN), San Diego, under Contract No. N66001-06-C-2020 (CBMANET).

\section{References}

[Bass et al. 03] A. Bass, C. Clark, A. Simmons, R. Fay, and A. Popper. "The Physical Acoustics of Underwater Sound Communication." In Acoustic Communication, Springer Handbook of Auditory Research 16, pp. 15-64. Springer, 2003.

[Chapeau-Blondeau and Monir 02] F. Chapeau-Blondeau, and A. Monir. "Numerical Evaluation of the Lambert W Function and Application to Generation of Generalized Gaussian Noise With Exponent 1/2." IEEE Trans. on Signal Proc. 50:9 (2002), 21602165.

[Franceschetti et al. 07] M. Franceschetti, O. Dousse, D. N. C. Tse, and P. Thiran. "Closing the Gap in the Capacity of Wireless Networks via Percolation Theory." IEEE Trans. Inf. Theory 53:3 (2007), 1009-1018.

[Grossglauser and Tse 02] M. Grossglauser and D. N. C. Tse. "Mobility Increases the Capacity of Ad Hoc Wireless Networks." IEEE Trans. Inf. Theory 10:4 (2002), 477486.

[Gupta and Kumar 00] P. Gupta and P. R. Kumar. "The Capacity of Wireless Networks." IEEE Trans. Inf. Theory 46:2 (2000), 388-404.

[Lucani et al. 08a] D. E. Lucani, M. Médard, and M. Stojanovic. "Capacity Scaling Laws for Underwater Networks." In Proc. 42nd Asilomar Conference on Signals, Systems and Computers, pp. 2125-2129, Pacific Grove, CA, USA, October 2008.

[Lucani et al. 08b] D. E. Lucani, M. Médard, and M. Stojanovic. "Underwater Acoustic Networks: Channel Models and Network Coding Based Lower Bound on Transmission Power for Multicast." IEEE Journal on Selected Areas in Communications (JSAC), Special Issue on Underwater Wireless Communications and Networks, pp. 1708-1719, December 2008.

[Ozgur et al. 07] A. Ozgur, O. Leveque, and D. N. C. Tse. "Hierarchical Cooperation Achieves Optimal Capacity Scaling in Ad Hoc Networks." IEEE Trans. Inf. Theory 53:10 (2007), 3549-3672. 
[Stojanovic 07] M. Stojanovic. "On the Relationship between Capacity and Distance in an Underwater Acoustic Communication Channel." ACM SIGMOBILE MC2R 11:4 (2007), 34-43.

[Vu et al. 08] M. Vu, N. Devroye, and V. Tarokh. "An Overview of Scaling Laws in Ad Hoc and Cognitive Radio Networks." Wireless Pers. Comm. 45:3 (2008), 343-354.

Daniel E. Lucani, Department of Electronic Systems, Aalborg University, Fredrik Bajers Vej 7, A3-205, 9220, Aalborg Øst, Denmark (del@es.aau.dk)

Muriel Médard, RLE, Massachusetts Institute of Technology, 77 Massachusetts Avenue, Room 36-512F, Cambridge, MA 02139, USA (medard@mit.edu)

Milica Stojanovic, Northeastern University, 409 Dana, Boston, MA 02115, USA (millitsa@mit.edu) 